\title{
Performance analysis of user association strategy based on power-domain non-orthogonal multiple access in visible light communication multi-cell networks
}

\author{
Siyu Tao, Hongyi Yu*, Qing Li and Yanqun Tang
}

\begin{abstract}
A multi-cell visible light communication (VLC) network with non-orthogonal multiple access (NOMA) introduces an inter-cell interference $(\mathrm{ICI})$, which causes poor experience of overlap-area user association. In this study, we focus on the strategic analysis of user association in multi-cell NOMA-VLC networks and reveal the relationship between the performance of user association and visible-light cell deployment. First, we establish the model of power-domain NOMA in multiple cells of VLC. Second, when the principle of proximity access (PPA) considers channel attenuation, we present the violation of the principle of proximity access (VPPA) based on signal-to-interference-plus-noise ratio (SINR) and derive a closed-form sufficient condition for overlap-area users based on the visible light channels. Third, based on the sufficient condition, we perform the strategic analysis of user association in two cases, i.e., the scenario probability with different numbers of users and the probability of the ratio of channel gains with an edge user are deduced. In addition, we evaluate the performance of user association by the deployment of lighting angles and inter-cell distance. Finally, the simulation results indicate that the VPPA achieves better data rate performance than the PPA based on the sufficient condition. Furthermore, the probability distributions in numerical results show that the situations with the VPPA reach a certain scale of occurrence probability.
\end{abstract}

Keywords: Visible light communication, Non-orthogonal multiple access, Multiple cells, User association, Cell deployment, Principle of proximity access, Probability distribution

\section{Introduction}

Visible light communication (VLC) [1] is an optical wireless communication technology for propagating the radio wave within the visible optical spectrum. In the next generation of wireless communication networks, a VLC network with non-orthogonal multiple access (NOMA) $[2,3]$ can be regarded as a useful enhancement which can

\section{*Correspondence: xxgcmaxyu@163.com}

This work was presented in part at the IEEE 19th International Conference on Communication Technology (ICCT 2019), China. The associate editor coordinating the review of this paper approved this complementary and revised paper for publication.

National Digital Switching System Engineering and Technological Research Center (NDSC), 450001 Zhengzhou, People's Republic of China help handle the traffic density $[4,5]$, equipment density, and user connection density $[6,7]$.

However, there are several challenges [8] of user association in NOMA-VLC networks. VLC networks perform line-of-sight (LOS) propagation of light to form directional coverage, which is a smaller cell compared with cellular radio frequency (RF) [1]. Owing to increasing inter-cell interference (ICI) in small VLC cells, optimizing transmit or receive strategies for multi-cell NOMA networks remains rather challenging [7]. Imperfect channel state information (CSI) that exists in the received signals is introduced by both ICI and NOMA, which may be treated as interference or effective signals regarding to signal processing schemes. In summary, user association, 
particularly the association of edge users within overlaparea users, focuses on the signal quality or received signal condition, which is affected by networking scenario, such as imperfect CSI and channel model.

Other than the traditional orthogonal multiple access (OMA) schemes, we have focused on other schemes, solving the multi-cell user association. On the one hand, enhanced multiple access schemes, such as the hybrid power domain sparse code multiple access $[9,10]$, guarantee that the users in overlap area can demodulate the signal or improve the signal quality, where these enhanced schemes attempt to fundamentally eliminate the ICI in the overlap area. On the other hand, distributed resource allocation schemes are developed [11-14], where interference mitigation is performed by the power allocation. Furthermore, multi-cell techniques such as coordinated scheduling and joint processing can be introduced to find optimum strategies [7]. For coordinated scheduling, another study [15] reviewed the current research that used VLC access point (AP) cooperation techniques for improving VLC networks and mitigate interferences. For joint processing, both joint transmission (JT) $[8,9,16,17]$ and dynamic cell selection (DCS) techniques have been studied [7]. From the above, user association in NOMA-VLC [18] is an important base for these multi-cell techniques; traditional proximity access principles [16, 19-21] favor the move towards the proximity of a transmitting light emitting diode (LED). In addition, the principles of user association can be evaluated based on the received signal strength [22] and signal-to-noise ratio [17, 23, 24]. Therefore, the interference by multi-cell NOMA might be complex and unexpected in different user groups or channel orders $[14,25]$, leading to difference in performance of proximity access principles.

This paper focuses on the performance analysis of edge user association strategy in a NOMA-VLC network without the decrease of central user experience. When a common transmission mode $[7,26]$ is assumed in a way that user data are shared among multiple APs but are transmitted only from a selected AP, the principle of proximity access (PPA) considers the minimum channel attenuation [27-30], which is strongly correlated with the minimum distance [28-30] between VLC transmitters and receivers. However, upon applying power-domain NOMA (PDNOMA) for multiple cells, a VLC network may encounter different levels of interference, leading to the weak performance of the traditional proximity access principle for edge users within overlap areas. In this paper, we aim towards the performance analysis in violation of the principle of proximity access (VPPA) for the edge users within the overlap area of a multi-cell NOMA-VLC network. First, we establish the system model for an indoor multicell VLC network and explain ICI expressions in multi-cell NOMA. Second, we analyze the strategy of user access schemes and present the violation of the principle of proximity access. Then, we derive a closed-form sufficient condition to support the violation. Third, combined with the sufficient condition, we study the cell deployment of a multi-cell NOMA-VLC network in two cases. In case 1, it indicates the possibility of the expected occurrence, while we analyze the scenario probability based on visible-light channels and the number of users. In case 2, the probability density distribution (PDF) of the ratio of channel gains is discussed, where it is a primary factor in the sufficient condition and it involves with the parameters of lighting deployment. In both the cases, we can observe the performance of the violation of the principle of proximity access with lighting deployment. Finally, we perform a simulation to explain the situation of the violation of the principle of proximity access. The gain and probability performances of the violation of the traditional access principle have been observed, which is consistent with the theoretical analysis.

\section{System model}

\subsection{Indoor VLC channel}

The overall coverage of a single AP LED at the ceiling of a room is defined as a cell of the VLC network. Users within the cell use the photo diode as a VLC signal receiver. The indoor VLC signal model can be referred to as a Lambertian radiation model [31]. Considering that the VLC signal in the LOS path is the main energy component in Fig. 1, we denote the VLC channel gain for the $k$-th user as shown in (1).

$$
h_{k}=\frac{(m+1) A}{2 \pi d_{k}^{2}} \cdot \cos ^{m}\left(\phi_{k}\right) \cdot T_{f} \cdot g\left(\psi_{k}\right) \cdot \cos \left(\psi_{k}\right),
$$

where $m$ is the order of Lambertian emission, $A$ is the physical area of the detector, $d_{k}$ is the distance between an LED transmitter and a receiver of the $k$ th user, $\phi_{k}$ is the angle of irradiance for the $k$ th user, $\psi_{k}$ is the angle of incidence for the $k$ th user, $T_{f}$ is the constant for an optical filter gain, and $g\left(\psi_{k}\right)$ is the gain of an optical concentrator of the $k$ th user.

In addition, $m$ and $g\left(\psi_{k}\right)$ have been provided by (2) and (3), respectively. $\Phi_{1 / 2}$ is the semi-angle of the LED, $\Psi_{\mathrm{FOV}}$ is the width of the angle field of vision (FOV) at the receiver, and $n$ is a constant of the refractive index.

$$
\begin{aligned}
m & =-\frac{1}{\log _{2}\left(\cos \left(\Phi_{1 / 2}\right)\right)} \\
g\left(\psi_{k}\right) & = \begin{cases}\frac{n^{2}}{\sin ^{2}\left(\Psi_{\mathrm{FOV}}\right)}, & 0 \leq \psi_{k} \leq \Psi_{\mathrm{FOV}} \\
0 & , \psi_{k}>\Psi_{\mathrm{FOV}}\end{cases}
\end{aligned}
$$

For the $k$ th user, the received electrical signal power $P_{r}$ is derived from the transmitted optical signal power $P_{t}$ and optical-electrical conversion efficiency constant $r_{o e}$. Note that $h_{\mathrm{LED}, k}$ is the channel gain from the LED to the $k$ th 


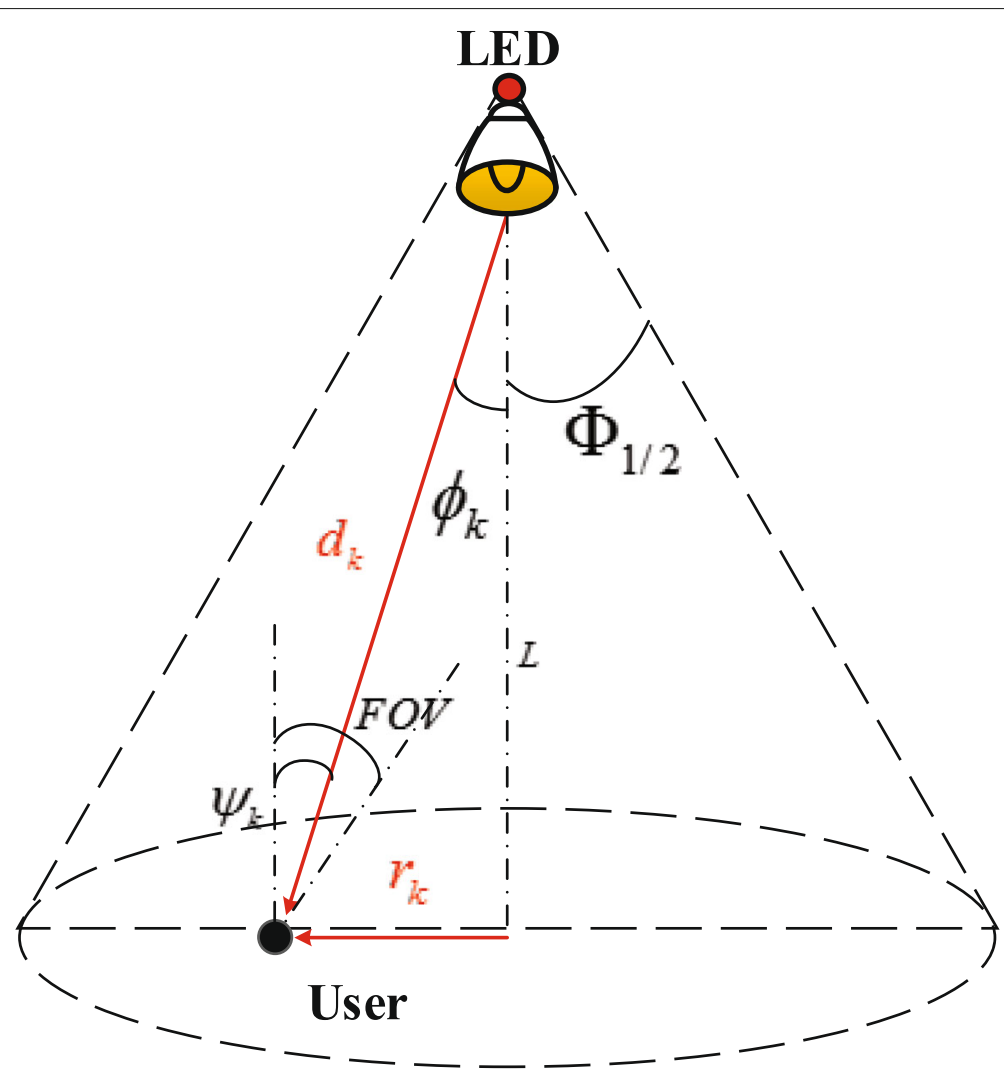

Fig. 1 VLC channel model

user, and $a_{\mathrm{LED}, k}$ is a power allocation coefficient controlling the transmitted signal power from the LED to the $k$ th user.

$$
P_{r}=r_{o e} \cdot h_{\mathrm{LED}, k} \cdot a_{\mathrm{LED}, k} \cdot P_{t}
$$

\subsection{Multi-cell PD-NOMA}

This paper emphasizes on multiple cells of NOMA-VLC networks and focuses on the experience of users in the overlap area (Fig. 2). If only PD-NOMA is adopted in multiple cells, the receivers in the overlap area may encounter imperfect CSI, as the overlap-cell user connected to LED $i$ (Fig. 2) may receive uncertain superposed signals from LED $j$. According to PD-NOMA,

Let us assume that $\tilde{u}$ is a set of users and $\tilde{u} \supseteq\{\tilde{\alpha}, \tilde{\beta}, \tilde{\gamma}\}$, where $\tilde{\gamma}$ indicates the set of users in the overlap cell and $\tilde{\alpha}, \tilde{\beta}$ indicates the set of users in the non-overlap cells. To clearly explain the derivation, we define several operators in (5)-(7), where the details are listed as follows.

- If $P_{t}$ is normalized, we define (5) to calculate the sum of the signal power coefficients of users, where the channel gain of the users is better than that of user $u^{*}, u^{*} \in \tilde{u}$. For example, $\tilde{u}=\left\{u_{1}, u_{2}, u_{3}, u_{4}, u_{5}\right\}, \tilde{\alpha}=$

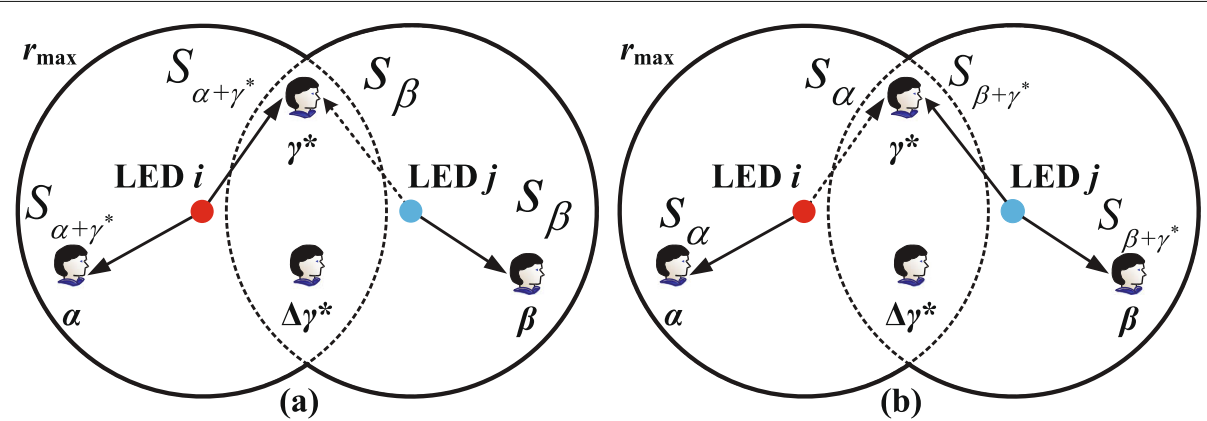

Fig. 2 Edge users within an overlap area in a multi-cell scenario 
$\left\{u_{1}, u_{2}, u_{3}\right\}, u_{4} \in \tilde{\gamma}, u_{5} \in \tilde{\beta}$. Within the same coverage of an LED, the users $\left\{u_{1}, u_{2}, u_{3}\right\}$ perform NOMA, where $h_{1}>h_{2}>h_{3}$ and $a_{1}<a_{2}<a_{3}$ are assumed. Finally, $\Gamma_{\mathrm{LED}}\left(\tilde{\alpha} \succ u_{3}\right)=a_{1}^{2}+a_{2}^{2}$. In addition, if $\left\{u_{1}, u_{2}, u_{3}, u_{4}\right\}$ are considered in the coverage of an LED and $h_{1}>h_{2}>h_{3}>h_{4}$ with $a_{1}<a_{2}<a_{3}<a_{4}$ is assumed,

$$
\begin{aligned}
& \Gamma_{\mathrm{LED}}\left(\tilde{\alpha}, \tilde{\gamma} \succ u_{4}\right)=a_{1}^{2}+a_{2}^{2}+a_{3}^{2} \text {. } \\
& \Gamma_{\mathrm{LED}}\left(\tilde{u} \succ u^{*}\right) \triangleq \sum_{\left\{u_{i} \mid h_{\mathrm{LED}, u_{i}}>h_{\mathrm{LED}, u^{*}}\right\}}\left(a_{\mathrm{LED}, u_{i}}\right)^{2}
\end{aligned}
$$

- We define (6) to calculate the number of users with better channel gain than that of a user. For example, if $\left\{u_{1}, u_{2}, u_{3}, u_{4}\right\}$ are considered in the coverage of an LED and $h_{1}>h_{2}>h_{3}>h_{4}$ with $a_{1}<a_{2}<a_{3}<a_{4}$ is assumed, $|\tilde{\alpha}, \tilde{\gamma} \succ 0|_{\mathrm{LED}}=5$ and $\left|\tilde{\alpha}, \tilde{\gamma} \succ u_{4}\right|_{\mathrm{LED}}=3$.

$$
\left|\tilde{u} \succ u^{*}\right|_{\mathrm{LED}} \triangleq \operatorname{card}\left\{u_{i} \mid h_{\mathrm{LED}, u_{i}}>h_{\mathrm{LED}, u^{*}}\right\}
$$

- We define the operation $\doteq$ in (7) to indicate that the local part of the objective function uniquely decides the positive and negative of the objective function. For example, if $f(h, a)=\frac{\left(h_{2} a_{2}\right)^{2}-\left(h_{1} a_{1}\right)^{2}}{\left(h_{1} a_{1}\right)^{2}\left(h_{2} a_{2}\right)^{2}}$ and $g(h, a)=\left(h_{2} a_{2}\right)^{2}-\left(h_{1} a_{1}\right)^{2}$, it is clear that $g(h, a)$ uniquely decides the positive and negative values of $f(h, a)$. Finally, if we want to prove $f(h, a) \geq 0$, we can prove $g(h, a) \geq 0$, when $f(h, a) \doteq g(h, a)$.

$$
\underbrace{f(h, a) \doteq g(h, a)}_{\text {Problem }} \Leftrightarrow \underbrace{g(h, a) \rightarrow 0_{-}^{+} \Rightarrow f(h, a) \rightarrow 0_{-}^{+}}_{\text {Equivalence Problem }}
$$

In Fig. 2a, user $\gamma_{*}$ from the overlap-cell user group $\tilde{r}$ has two choices, accessing either LED $i$ or LED $j$. The signalto-interference-plus-noise ratio (SINR) in two choices are different. If user $\gamma_{*}$ is connected to LED $i$, we can yield (8), where $i$ and $j$ denote LED $i$ and LED $j$, respectively, and $\operatorname{SINR}_{\gamma_{*} \in \tilde{\gamma}}^{i}$ denotes the SINR of user $\gamma_{*}$ connected to the channel of LED $i$. Note that the noise $\sigma^{2}=N_{0} B$ caused by different independent sources can be regarded as an additive white Gaussian noise, where $N_{0}$ is the constant noise power spectral density and $B$ is the constant bandwidth.

$$
\begin{aligned}
& \operatorname{SINR}_{\gamma_{*} \in \tilde{\gamma}}^{i}=\frac{h_{i, \gamma_{*}}^{2} a_{i, \gamma_{*}}^{2}}{\underbrace{h_{i, \gamma_{*}}^{2} \Gamma_{i}\left(\tilde{\alpha}, \tilde{\gamma} \succ \gamma_{*}\right)}_{\text {LED } i \text { (connected) }}+\underbrace{h_{j, \gamma_{*}}^{2} \Gamma_{j}(\tilde{\beta} \succ 0)}_{L E D j \text { (connectless) }}+\sigma^{2}}(8) \\
& \operatorname{SINR}_{\gamma_{*} \in \tilde{\gamma}}^{j}=\frac{h_{j, \gamma_{*}}^{2} a_{j, \gamma_{*}}^{2}}{\underbrace{h_{i, \gamma_{*}}^{2} \Gamma_{i}(\tilde{\alpha} \succ 0)}_{\text {LED } i \text { (connectless) }}+\underbrace{h_{j, \gamma_{*}}^{2} \Gamma_{j}\left(\tilde{\gamma}, \tilde{\beta} \succ \gamma_{*}\right)}_{\text {LED } j \text { (connected) }}+\sigma^{2}}(9)
\end{aligned}
$$

\section{NOMA-VLC user association in two cases}

\subsection{User groups in overlap area}

$$
\begin{aligned}
\operatorname{SINR}_{\gamma^{*} \in \tilde{\gamma}}^{j}-\operatorname{SINR}_{\gamma^{*} \in \tilde{\gamma}}^{i} \doteq & h_{i, \gamma^{*}}^{2} h_{j, \gamma^{*}}^{2}\left[a_{j, \gamma^{*}}^{2} \Gamma_{i}\left(\tilde{\alpha}, \tilde{\gamma} \succ \gamma^{*}\right)\right. \\
& \left.-a_{i, \gamma^{*}}^{2} \Gamma_{j}\left(\tilde{\beta}, \tilde{\gamma} \succ \gamma^{*}\right)\right] \\
& +h_{j, \gamma^{*}}^{4} a_{j, \gamma^{*}}^{2} \Gamma_{j}(\tilde{\beta} \succ 0) \\
& -h_{i, \gamma^{*}}^{4} a_{i, \gamma^{*}}^{2} \Gamma_{i}(\tilde{\alpha} \succ 0) \\
& +\sigma^{2} \cdot\left[h_{j, \gamma^{*}}^{2} a_{j, \gamma^{*}}^{2}-h_{i, \gamma^{*}}^{2} a_{i, \gamma^{*}}^{2}\right]
\end{aligned}
$$

For one user group in the overlap area, let us assume that the user $\gamma_{*}$ within the user group has a greater channel gain given by LED $i$, which is nearer to the user (i.e.,

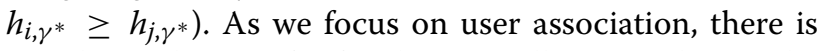
a realistic chance of a fixed power allocation during the initial process of user association [32]. If the allocation is done, the principle of proximity access would then indicate that the user should be connected to LED $i$. However, because of the different interferences caused by multi-cell PD-NOMA for each edge user, the principle of proximity access may not be available. It means that the situation where the user group nearer to LED $i$ connects to LED $j$ may exist in a non-negative (10).

When the quality of the received signal in (10) is focused, the purpose for the violation of proximity access is involved with edge or overlap-cell user association. These complex factors jointly affect the positive and negative values, including the comparisons in $h, a, \Gamma$. Due to the difficulty of closed form analysis, we yield two cases to analyze the violation of the proximity access. On the one hand (i.e., in case 1), we simplify the comparison with fixed power allocation and realize the condition for violation of the principle of proximity access. On the other hand (i.e., in case 2), based on visible-light channel, we derive the cell deployment with the number of user and the probability of the violation of proximity access, when fixed power allocation is assumed. Hence, it is indicated via two cases as follows.

\subsection{Sufficient condition based on visible-light channels}

With the aforementioned assumption, if user $\gamma^{*}$ can receive better signal quality with respect to LED $j$ at a greater distance, (9) would require a positive value, leading to the violation of the principle of proximity access. When a uniform power allocation, that is one of the schemes of fixed power allocation, is done, a positive condition is established in (10). Hence, the compact sufficient condition that guarantees $Q_{1}, Q_{2}, Q_{3} \geq 0$ can be indicated in (11). 


$$
\begin{aligned}
& \operatorname{SINR}_{\gamma^{*} \in \tilde{\gamma}}^{j}-\operatorname{SINR}_{\gamma^{*} \in \tilde{\gamma}}^{i} \\
& \doteq \underbrace{\frac{h_{i, \gamma^{*}}^{2} h_{j, \gamma^{*}}^{2}}{(|\tilde{\gamma}|+|\tilde{\alpha}|)^{2}(|\tilde{\gamma}|+|\tilde{\beta}|)^{2}}\left[\left|\tilde{\alpha}, \tilde{\gamma} \succ \gamma^{*}\right|_{i}-\left|\tilde{\beta}, \tilde{\gamma} \succ \gamma^{*}\right|_{j}\right]}_{Q_{1}} \\
& +\underbrace{\frac{h_{j, \gamma^{*}}^{4}}{(|\tilde{\gamma}|+|\tilde{\beta}|)^{2}|\tilde{\beta}|^{2}}|\tilde{\beta} \succ 0|_{j}-\frac{h_{i, \gamma^{*}}^{4}}{(|\tilde{\gamma}|+|\tilde{\alpha}|)^{2}|\tilde{\alpha}|^{2}}|\tilde{\alpha} \succ 0|_{i}}_{Q 2} \\
& +\underbrace{\sigma^{2}\left[\frac{h_{j, \gamma^{*}}^{2}}{(|\tilde{\gamma}|+|\tilde{\beta}|)^{2}}-\frac{h_{i, \gamma^{*}}^{2}}{(|\tilde{\gamma}|+|\tilde{\alpha}|)^{2}}\right]}_{Q 3} \\
& 1 \leq\left(\frac{h_{i, \gamma^{*}}}{h_{j, \gamma^{*}}}\right)^{2} \leq \min \left\{\left(\frac{|\tilde{\gamma}|+|\tilde{\alpha}|}{|\tilde{\gamma}|+|\tilde{\beta}|}\right) \cdot \sqrt{\frac{|\tilde{\alpha}|}{|\tilde{\beta}|}},\left(\frac{|\tilde{\gamma}|+|\tilde{\alpha}|}{|\tilde{\gamma}|+|\tilde{\beta}|}\right)^{2}\right\}
\end{aligned}
$$

For a newly added user group in the overlap area, let us assume that two types of user groups, $\tilde{\gamma}$ and $\Delta \tilde{\gamma}$, are in the overlap area. If we focus on the newcomer $\Delta \tilde{\gamma}$ based on the existing connected user group $\tilde{\gamma}$, the received quality can be analyzed in (13). Considering $Q_{4}, Q_{5}, Q_{6} \geq 0$, we can apply the compact sufficient condition in (14), leading to the violation of the principle of proximity access.

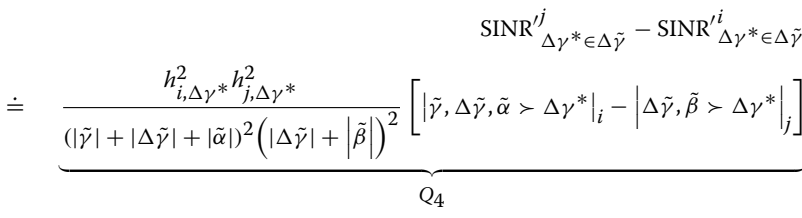

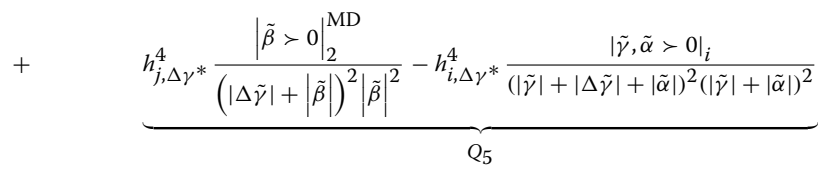

$$
\begin{aligned}
& +\underbrace{\sigma^{2}\left[\frac{h_{j, \Delta \gamma^{*}}^{2}}{(|\Delta \tilde{\gamma}|+|\tilde{\beta}|)^{2}}-\frac{h_{i, \Delta \gamma^{*}}^{2}}{(|\tilde{\gamma}|+|\Delta \tilde{\gamma}|+|\tilde{\alpha}|)^{2}}\right]}_{Q_{6}}
\end{aligned}
$$

$$
\begin{aligned}
1 \leq\left(\frac{h_{i, \Delta \gamma^{*}}}{h_{j, \Delta \gamma^{*}}}\right)^{2} \leq & \min \left\{\left(\frac{|\tilde{\gamma}|+|\Delta \tilde{\gamma}|+|\tilde{\alpha}|}{|\Delta \tilde{\gamma}|+|\tilde{\beta}|}\right) \cdot \sqrt{\frac{|\tilde{\gamma}|+|\tilde{\alpha}|}{|\tilde{\beta}|},}\right. \\
& \left.\left(\frac{|\tilde{\gamma}|+|\Delta \tilde{\gamma}|+|\tilde{\alpha}|}{|\Delta \tilde{\gamma}|+|\tilde{\beta}|}\right)^{2}\right\}
\end{aligned}
$$

According to the mathematical induction, based on the existing guaranteed user groups in the overlap area, we can consider that the current user group in the overlap area may not follow the principle of proximity access. When the number of users connected to LED $i$ and LED $j$ is $u$ and $v$, respectively, the current user group selects the violation of the principle of proximity access in (15), where the number of current users is $w$. Note that the left minimum condition is selected by $w \geq \sqrt{u \cdot v}$.

$$
1 \leq\left(\frac{h_{i, w}}{h_{j, w}}\right)^{2} \leq \min \left\{\left(\frac{w+u}{w+v}\right) \cdot \sqrt{\frac{u}{v}},\left(\frac{w+u}{w+v}\right)^{2}\right\}
$$

If we focus on the edge user based on visible-light channels in the overlap area, the strategy selection of the edge user for accessing AP can be affected by (15). If $d_{\text {LED, } k}$ is the distance between the LED and $k$ th user, we can yield $d_{\mathrm{LED}, k}=\sqrt{r_{\mathrm{LED}, k}^{2}+L^{2}}$, where $r_{\mathrm{LED}, k}$ is the radius of user location in Fig. 1. Therefore, in VLC multiple cells, the edge user who should abandon the principle of proximity access with minimum visible-light channel attenuation should consider the cell deployment in (16). Note that the condition to satisfy for selecting the VPPA affects the access of edge users in the sequential iteration, and thus, the user association strategy focused in this paper can be commonly used for grouping and handover of edge users.

$$
\begin{aligned}
1 & \leq \ln \left(\frac{r_{j, w}^{2}+L^{2}}{r_{i, w}^{2}+L^{2}}\right) \leq \frac{4}{m+3} \cdot \ln \left(\frac{w+u}{w+v}\right) \\
& =\frac{4}{3-\frac{1}{\log _{2}\left(\cos \left(\Phi_{1 / 2}\right)\right)}} \cdot \ln \left(\frac{w+u}{w+v}\right)
\end{aligned}
$$

The order of Lambertian emission $m$ is uniquely involved with $\Phi_{1 / 2}$ and is inversely proportional to the semi-angle. According to (15), the higher value of $\Phi_{1 / 2}$ facilitates the success of the sufficient condition. On the other hand, the proportion of the number of multi-cell users affects the strategy of user access. In summary, the aforementioned derivation is based on the visible light channel and is involved with the lighting parameters of cell deployment.

\subsection{Performance case 1: scenario probability with the number of users}

When other fixed power allocation schemes are focused, that are not limited to equal allocation. Several fixed power allocation schemes include power law scheme, fractional transmit power allocation, and improved fractional scheme, where the assigned power allocation factor decreases as the ratio of the channel gain increases. In PDNOMA, without loss of generality, the power allocation factors in those schemes are normalized. Hence, in (10), $\Gamma_{j}(\tilde{\beta} \succ 0)$ and $\Gamma_{i}(\tilde{\alpha} \succ 0)$ can be reduced.

If multiple cells independently adopt the same fixed power allocation scheme, which is not limited to equal allocation, the focus will be the non-negative first-item of (10). Namely, we hope $\Gamma_{i}\left(\tilde{\alpha}, \tilde{\gamma} \succ \gamma^{*}\right) \geq$ 
$\Gamma_{j}\left(\tilde{\beta}, \tilde{\gamma} \succ \gamma^{*}\right)$, which may form the $a_{j, \gamma^{*}}^{2} \geq a_{j, \gamma^{*}}^{2}$ during the same fixed power allocation scheme. Therefore, we focus on the probability of the expected scenario (i.e., the violation of proximity access) for sufficient conditions.

According to references [2, 3], the expression of visiblelight channel gain for $k$ th user can be transformed into (17). In (17), $\rho \triangleq \frac{A \cdot T_{f} \cdot g\left(\psi_{k}\right)}{2 \pi}$ and $r_{\max }$ is the maximum radius that can effectively receive the communication signal. Studied [2,3] have proved that the cumulative distribution function (CDF) of the unordered square of visiblelight channel $h_{k}^{2}$ for $k$ th user can be indicated in (18). In this case, we focus on the right part of (15), which reflects the effect of the number of users. If the number of users is $\left|u^{\prime}\right|$ in $\Gamma_{i}\left(\tilde{\alpha}, \tilde{\gamma} \succ \gamma^{*}\right)$ and $\left|v^{\prime}\right|$ in $\Gamma_{j}\left(\tilde{\beta}, \tilde{\gamma} \succ \gamma^{*}\right)$, this paper derives the expected probability event $\mathbb{P}_{\left|u^{\prime}\right| \geq\left|v^{\prime}\right|, h_{\gamma^{*}}^{2}}$, which means the least $\left|u^{\prime}\right|$ and no more $\left|v^{\prime}\right|$ in $P\left(h_{u^{\prime}}^{2} \geq h_{\gamma^{*}}^{2}\right)$. $P\left(h_{v^{\prime}}^{2} \leq h_{\gamma^{*}}^{2}\right)$. Hence, the probability of the expected scenario can be expressed in (19), where $\mathbb{C}_{\mathrm{A}}^{\mathrm{B}}$ denotes $\frac{\mathrm{A} !}{\mathrm{B} !(\mathrm{A}-\mathrm{B}) !}$.

$$
\begin{gathered}
h_{k}=\frac{\rho(m+1) L^{m+1}}{\left(r_{k}^{2}+L^{2}\right)^{\frac{m+3}{2}}} \\
F_{h_{k}^{2}}(t)=P\left(h_{k}^{2} \leq t\right) \\
\quad=-\frac{1}{r_{\max }^{2}}\left[\rho(m+1) L^{m+1}\right]^{\frac{2}{m+3}} t^{-\frac{1}{m+3}}+\frac{L^{2}}{r_{\max }^{2}}+1
\end{gathered}
$$

$$
\begin{aligned}
\mathbb{P}_{\left|u^{\prime}\right| \geq\left|\nu^{\prime}\right|, h_{\gamma^{*}}^{2}} & {\left[\sum_{i=\left|u^{\prime}\right|}^{|\tilde{\alpha}|} \mathbb{C}_{|\tilde{\alpha}|}^{i} \cdot F_{h_{k}^{2}}^{|\tilde{\alpha}|-i}\left(\gamma^{*}\right) \cdot\left(1-F_{h_{k}^{2}}\left(\gamma^{*}\right)\right)^{i}\right] } \\
& \cdot\left[\sum_{j=|\tilde{\beta}|-\left|\nu^{\prime}\right|}^{|\tilde{\beta}|} \mathbb{C}_{|\tilde{\beta}|}^{j} \cdot F_{h_{k}^{2}}^{j}\left(\gamma^{*}\right) \cdot\left(1-F_{h_{k}^{2}}\left(\gamma^{*}\right)\right)^{|\tilde{\beta}|-j}\right]
\end{aligned}
$$

\subsection{Performance case 2: PDF of the channel gain ratio for edge user}

In this case, we focus on the ratio between the channel gains, which is the middle part of (15). If a user exists in the overlap or edge cell, we focus on the probability of the successful condition in (15).

First, let us assume that an edge user within overlap areas has two connections, which yield two channel gains $h_{i}$ and $h_{j}$. Based on (17), the ratio between channel gains (i.e., $\stackrel{\circ}{h}$ ) denotes $\frac{h_{i}}{h_{j}}$ in (20). In addition, $d_{\text {LED }}$ refers to the distance between two LEDs, $r_{x}$ is the radius of the user connecting to the left LED. Without loss of generality in Fig. 3, we assume that the location of two cells is related to $r_{\max } \leq d_{\mathrm{LED}} \leq 2 r_{\max }$ and the maximum radius of the coverage of two cells is the same.

$$
\begin{aligned}
\stackrel{\circ}{h}=\frac{h_{i}}{h_{j}}=\frac{\left(r_{j}^{2}+L^{2}\right)^{\frac{m+3}{2}}}{\left(r_{i}^{2}+L^{2}\right)^{\frac{m+3}{2}}} & =\left[\frac{\left(d_{\mathrm{LED}}-r_{x}\right)^{2}+L^{2}}{r_{x}^{2}+L^{2}}\right]^{\frac{m+3}{2}} \\
= & {\left[1+\frac{d_{\mathrm{LED}}^{2}-2 d_{\mathrm{LED}} r_{x}}{r_{x}^{2}+L^{2}}\right]^{\frac{m+3}{2}} }
\end{aligned}
$$

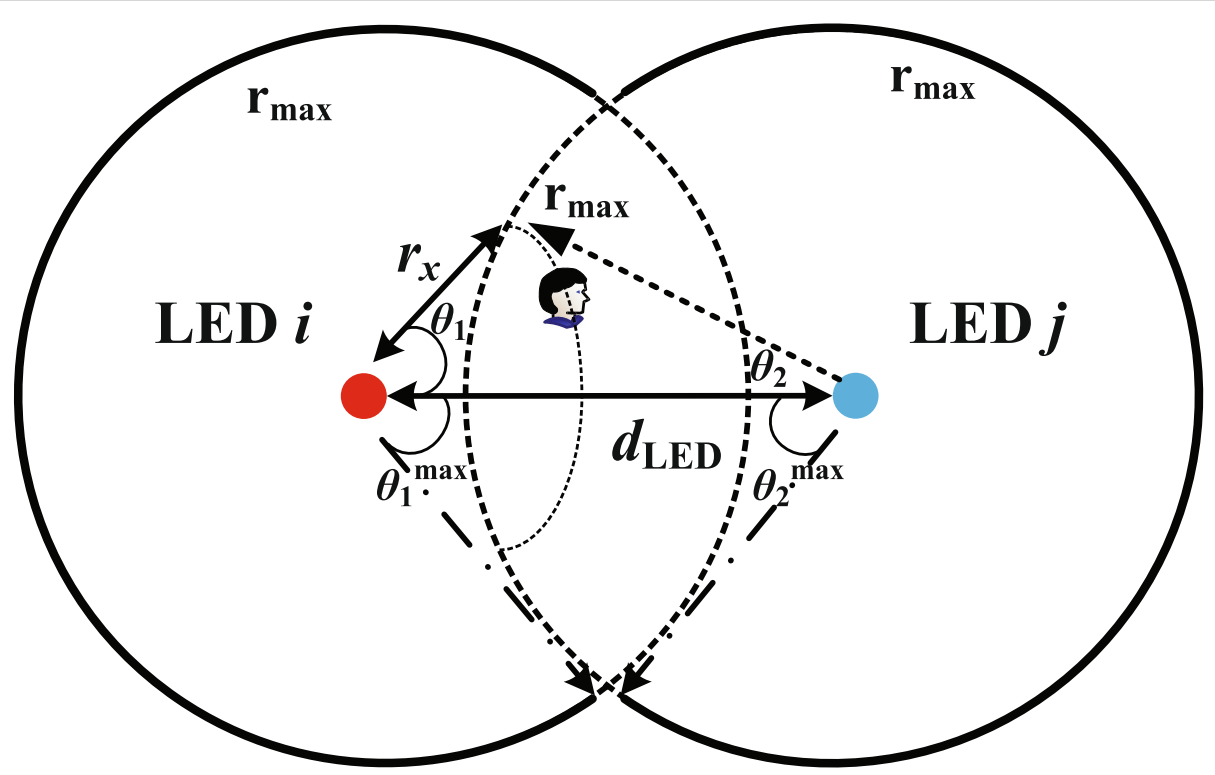

Fig. 3 Geometrical parameters of an edge user within the overlap area 


$$
\begin{aligned}
& r_{x}=\frac{-2 d_{\mathrm{LED}}+\sqrt{4 d_{\mathrm{LED}}^{2}-4 \xi^{2} L^{2}+4 \xi d_{\mathrm{LED}}^{2}}}{2 \xi} \\
& \xi \triangleq e^{\frac{2}{m+3} \cdot \ln \left({ }^{\circ}\right)}-1 \equiv \frac{d_{\mathrm{LED}}^{2}-2 d_{\mathrm{LED}} r_{x}}{r_{x}^{2}+L^{2}}
\end{aligned}
$$

Second, the function $\stackrel{\circ}{h}\left(r_{x}\right)$ is given in (20). If the function $G^{-1}(\bullet)$ means the inverse function of $\stackrel{\circ}{h}\left(r_{x}\right)$, we can yield $r_{x}=G^{-1}\left(\begin{array}{l}\circ \\ h\end{array}\right)$ in (21), where $\xi$ is indicated in (22). When the lighting deployment is determined, the unique independent variable is $h$, and $\xi$ in (22) can be regarded as a function $\xi(\stackrel{\circ}{h})$. Afterwards, we yield $\frac{\partial \stackrel{\circ}{\partial} r_{x}}{\text { in }}$ (23) for the calculation of the PDF of $f(\stackrel{\circ}{h})$.

$$
\begin{array}{r}
\frac{\partial \stackrel{\circ}{h}}{\partial r_{x}}=\frac{m+3}{2}[1+\xi]^{\frac{m+1}{2}} \frac{\partial \xi}{\partial r_{x}}=\frac{m+3}{2}[1+\xi]^{\frac{m+1}{2}} \\
{\left[-\frac{2 d_{\mathrm{LED}}}{r_{x}^{2}+L^{2}}-\frac{\left(d_{\mathrm{LED}}^{2}-2 d_{\mathrm{LED}} r_{x}\right) 2 r_{x}}{\left(r_{x}^{2}+L^{2}\right)^{2}}\right]}
\end{array}
$$

Third, with references [2, 3], the PDF of $f(\stackrel{\circ}{h})$ can be derived in (24), where $f\left(r_{x}\right)$ means the PDF of the variable $r_{k}, \xi(\stackrel{\circ}{h})$ is indicated in (22), and $\xi^{\prime}(\stackrel{\circ}{h})$ can be expressed in (25).

$$
\begin{aligned}
& f(\stackrel{\circ}{h})=\left|\frac{\partial G^{-1}(\stackrel{\circ}{h})}{\partial \stackrel{\circ}{h}}\right| \cdot f\left(r_{x}\right)=\left|\frac{1}{\frac{\partial}{\partial h}}\right| \cdot f\left(r_{x}\right) \\
& =\left|\frac{m+3}{2}\left[1+\xi\left(\begin{array}{l}
\circ \\
h
\end{array}\right)\right]^{\frac{m+1}{2}}\left[-\frac{2 d_{\mathrm{LED}}}{r_{x}^{2}+L^{2}}-\frac{\left(d_{\mathrm{LED}}^{2}-2 d_{\mathrm{LED}} r_{x}\right) 2 r_{x}}{\left(r_{x}^{2}+L^{2}\right)^{2}}\right]\right|^{-1} \cdot f\left(r_{x}\right) \\
& =\left|\frac{m+3}{2}[1+\xi(\stackrel{\circ}{h})]^{\frac{m+1}{2}}\left[-\frac{2 d_{\mathrm{LED}}}{\xi^{\prime 2}(\stackrel{\circ}{h})+L^{2}}-\frac{\xi^{2}\left(\begin{array}{l}
\circ \\
h
\end{array}\right)}{\frac{d_{\mathrm{LED}}^{2}}{2 \xi^{\prime}(\stackrel{\circ}{\circ})}-d_{\mathrm{LED}}}\right]\right|^{-1} \cdot f\left(r_{x}\right)
\end{aligned}
$$

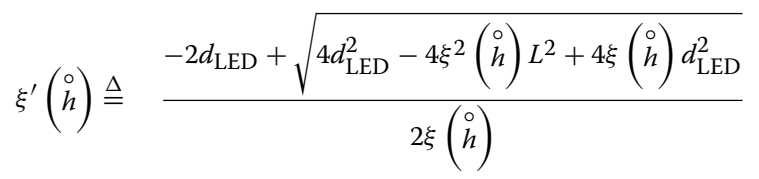

Furthermore, $f\left(r_{x}\right)$ can be indicated in (26), where $S_{e}$ is the area of the overlap cell. For example in Fig. $3, S_{e}=$ $\left(\theta_{1}^{\max }+\theta_{2}^{\max }\right) \cdot r_{\max }^{2}-r_{\max } \cdot d_{\mathrm{LED}} \cdot \sin \left(\theta_{1}^{\max }\right)$ and $\theta_{1}^{\max }, \theta_{2}^{\max }$ in radian measures are certain.
Finally, when (25) and (26) are introduced into (24), the unique independent variable is $\stackrel{\circ}{h}$, and then the PDF of $\stackrel{\circ}{h}$ can be expanded in (24). Note that when the lighting deployment and the overlap area are determined, the parameters except $h$ in (24), (25) and (26) are determined.

$$
\begin{aligned}
f\left(r_{x}\right) & =\frac{2 \operatorname{arcos}\left(\frac{r_{x}^{2}+d_{\mathrm{LED}}^{2}-r_{\max }^{2}}{2 r_{x} d_{\mathrm{LED}}}\right) \cdot r_{x}}{S_{e}} \\
& =\frac{2 \operatorname{arcos}\left(\frac{\xi^{\prime 2}\left(\begin{array}{c}
\circ \\
h
\end{array}\right)+d_{\mathrm{LED}}^{2}-r_{\max }^{2}}{2 \xi^{\prime}(\stackrel{\circ}{h}) d_{\mathrm{LED}}}\right) \cdot \xi^{\prime}\left(\begin{array}{l}
\circ \\
h
\end{array}\right)}{S_{e}}
\end{aligned}
$$

\section{Results and discussion}

As indicated in Table 1, the relevant simulation parameters are reported in $[2,3,31]$. The simulations are designed in two types: one type is to observe the system performance such as the user data rate, and the other one indicates the factors of cell deployment (including half-power semi-angles, scenario probability, and the probability of channel gain ratio) that affect the strategy of user association. The simulations were done using the Monte Carlo method to generate a uniform user distribution.

\subsection{Spectral efficiency of edge users}

Figure 4 shows that due to ICI, a coverage hole develops in the overlap areas of multiple cells. When the cell deployment requires a seamless coverage of illumination, users may exist in the coverage hole. When PD-NOMA or OMA without proper resource management is introduced, the multi-user interference (MUI) in the overlap area may increase; then, the interference in the overlap area will be more. Hence, the resource management, including strategies of user access, should be designed.

To improve the spectral efficiency, we use the NOMA to enhance the system throughput, while we dynamically select the strategy of user access to reduce the ICI and MUI. Let us denote PPA as the traditional principle of proximity access and VPPA as the violation of the principle of proximity access. If cell deployment is a regular

Table 1 Experimental parameters

\begin{tabular}{llll}
\hline Symbol & Value & Symbol & Value \\
\hline$r_{\text {oe }}$ & $0.53(\mathrm{~A} / \mathrm{W})$ & $\Psi_{\text {FOV }}$ & $60^{\circ}$ \\
$\Phi_{1 / 2}$ & $30^{\circ} \sim 60^{\circ}$ & $m$ & $4.8 \sim 1$ \\
$T_{f}$ & 1 & $n$ & 1.5 \\
$g\left(\psi_{k}=\Psi_{\text {FOV }}\right)$ & 3 & $L$ & $2.5(\mathrm{~m})$ \\
$N_{0}$ & $10^{-19}\left(\mathrm{~A}^{2} / \mathrm{Hz}\right)$ & $B$ & $20(\mathrm{MHz})$ \\
$P_{t}$ & $9(\mathrm{~W})$ & $A$ & $10^{-4}\left(\mathrm{~m}^{2}\right)$ \\
Room 1 & $18 \times 18 \times 2.5\left(\mathrm{~m}^{3}\right)$ & Room 2 & $12 \times 6 \times 2.5\left(\mathrm{~m}^{3}\right)$ \\
\hline
\end{tabular}


arrangement, we can consider two cells, which have an overall area of $12 \times 6\left(\mathrm{~m}^{2}\right)$, as a typical multi-cell scenario. Because of the existence of the channel order in the PD-NOMA, different channel gain orders might be considered. For example, the case of $\forall h_{\tilde{\alpha}, i}, h_{\tilde{\beta}, j} \geq \max h_{\tilde{\gamma}, \forall i, j}$ means that the edge users have the worst channel gain, where any user within non-overlap cells includes the channel gain better than the edge users within overlap areas. And the case of $\exists h_{\tilde{\alpha}, i}, h_{\tilde{\beta}, j} \leq \max _{\tilde{\gamma}, \forall i, j}$ means that not all users within non-overlap cells have a better channel gain than the edge users.

Afterwards, we obtain Fig. 5 with different channel orders to indicate that the VPPA will offer more spectral efficiency than PPA. As shown in Fig. 5, we evaluate spectral efficiency by obtaining the ratio of the average user data rate (AUDR) to bandwidth. Each user group has the same users leading to $\frac{w+u}{w+v} \approx 1$ and Monte Carlo simulation is performed in the uniform user distribution. Based on the cell deployment of (15), the VPPA improves the spectral efficiency of edge users. The VPPA also enhances the whole system's throughput to a certain extent. The strategy of user association actually realizes NOMA user grouping, which can uniformly decompose different interference components.

\subsection{Effect of cell deployment}

In cell deployment, the parameters involved with the semi-angle, number of users, the probability of scenario, and the probability of the ratio between channel gains are discussed in this simulation.

First, the relationship between the half-power semiangle $\Phi_{1 / 2}$ and the spectral efficiency is discussed. When semi-angle is $60^{\circ}$, the AUDR decreases (Fig. 6). However, the gain of AUDR in the VPPA is greater than that in the PPA. On the one hand, Fig. 6 shows the effect of the semiangle on the performance of multiple cells. On the other hand, we yield a supplementary simulation, as shown in Fig. 7, for a detailed explanation. Note that the incremental proportion is denoted as the enhancement ratio of the spectral efficiency difference between VPPA and PPA to the PPA.

According to the Fig. 6, the increased semi-angle will decrease the absolute value of the spectral efficiency, whereas for the users in overlap areas it is more appropriate to select the VPPA. However, we can discover the several-fold enhanced relative value of the spectral efficiency of edge users in Fig. 7. In Fig. 7, $|\tilde{\alpha}|$ and $|\tilde{\beta}|$ means the number of users in two cells, and the vertical axis reflects year-on-year growth ratio. Various users may generate different absolute values of spectral efficiency,

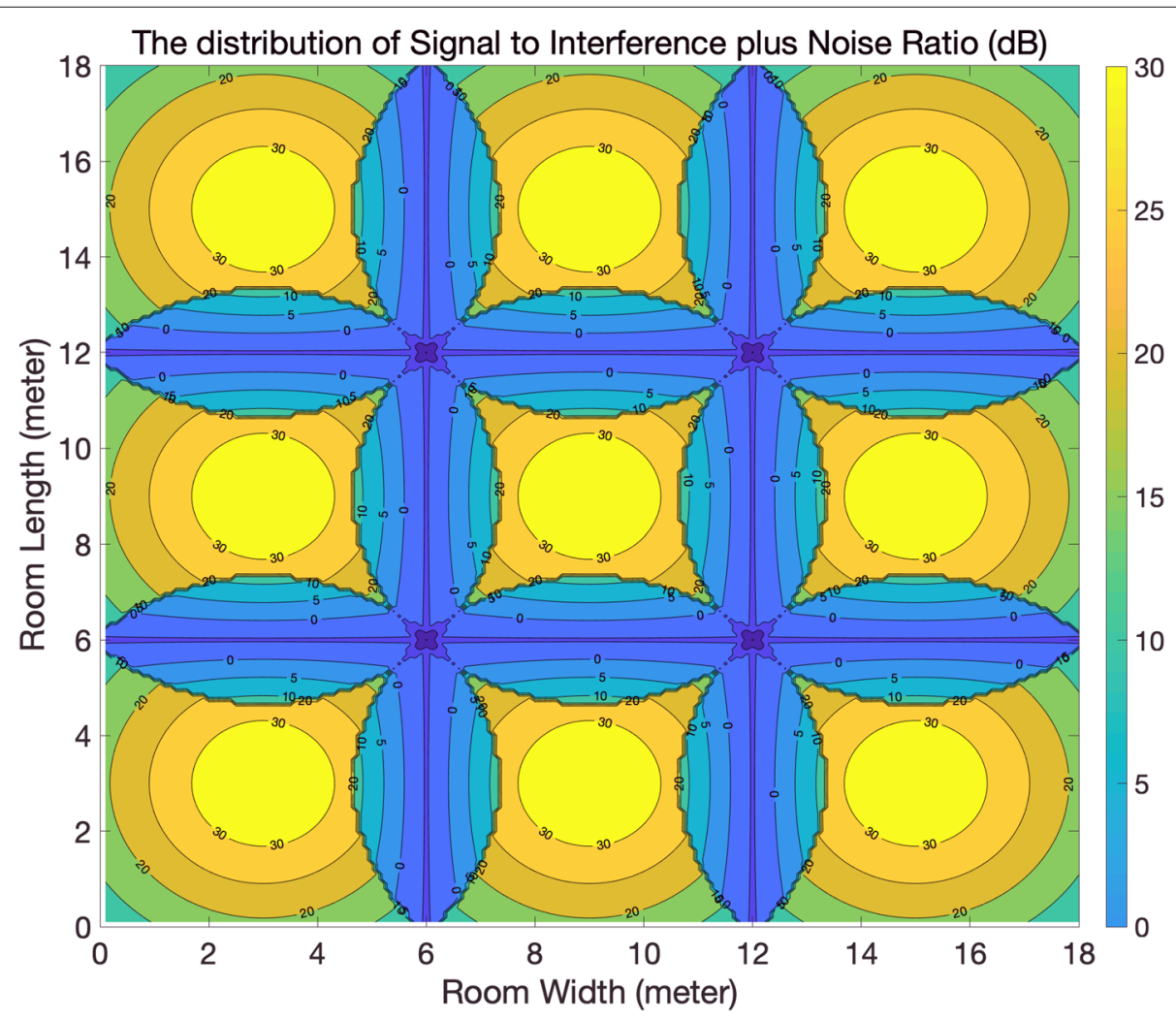

Fig. 4 SINR distribution in a multi-cell scenario 


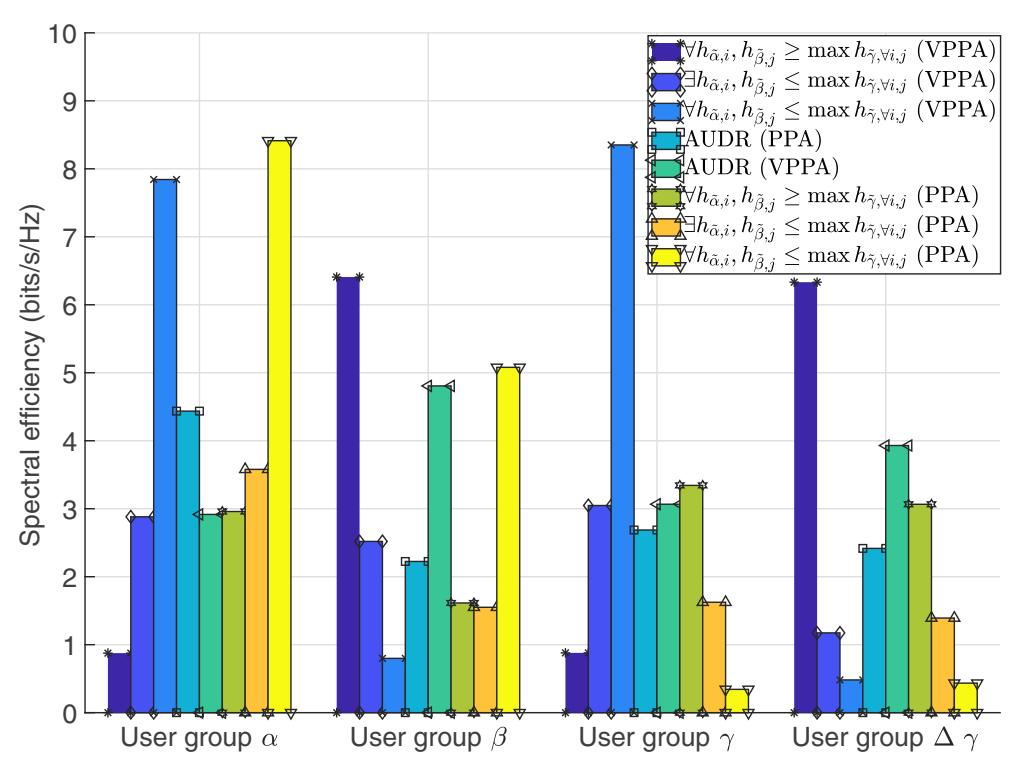

Fig. 5 Spectral efficiency of each user group with $\Phi_{1 / 2}=30^{\circ}$

whereas the similar ratio of the number of users has a similar performance, which can be indicated by (15).

Second, we observe the performance of channel gain distribution and scenario probability in Figs. 8 and 9. The channel gain distribution indicated in (17) shows that the distribution is mixed in the uniform distribution and the power law distribution. Hence, Fig. 8 has a similar trend, which includes a part of the straight slant, whereas the whole curves are not in the parabolic shape. Based on the channel gain distribution, in Fig. 9, we can analyze the scenario probability of the users selecting the strategy of violation of the principle of proximity access. If the expected scenario defines, the edge or overlap users may select the strategy of violation of the principle of proximity

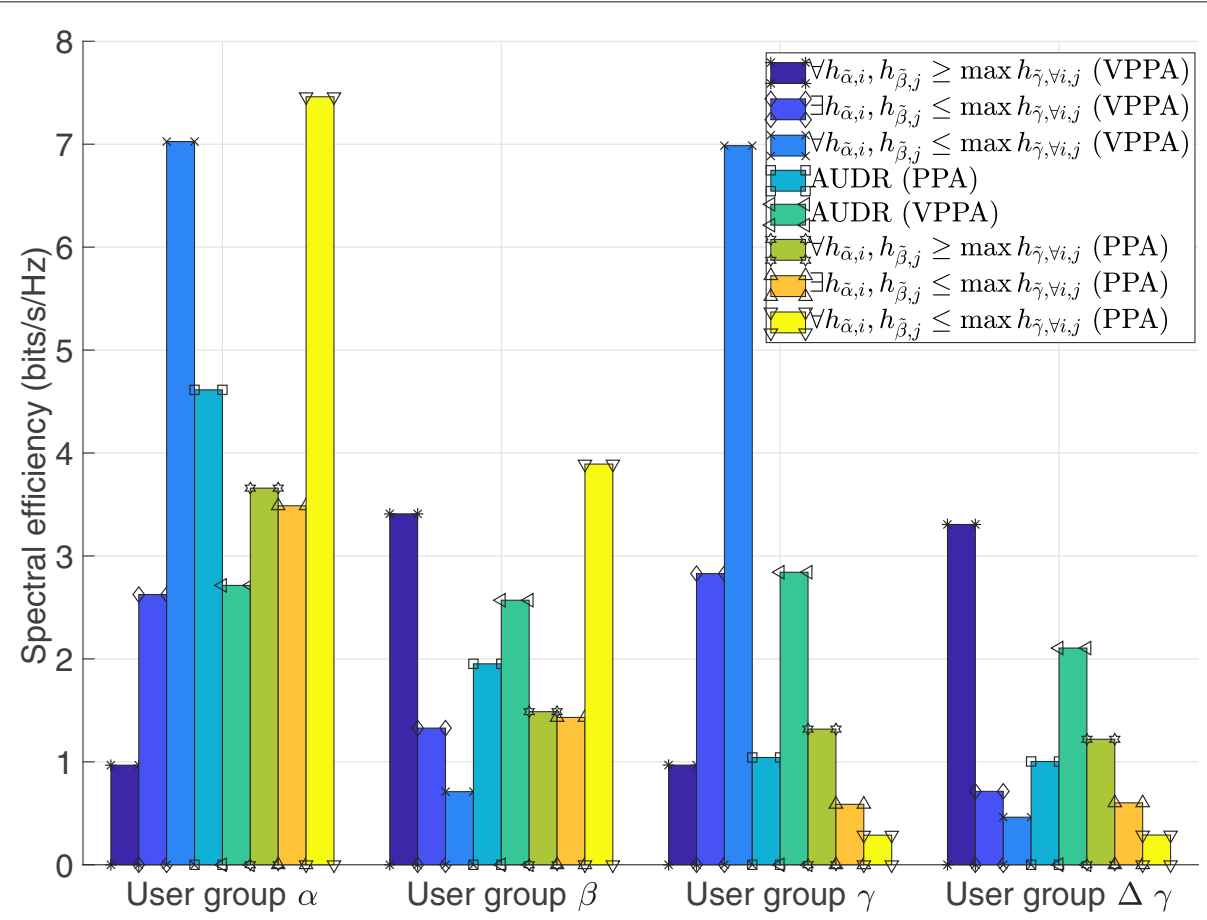

Fig. 6 Spectral efficiency of each user group with $\Phi_{1 / 2}=60^{\circ}$ 


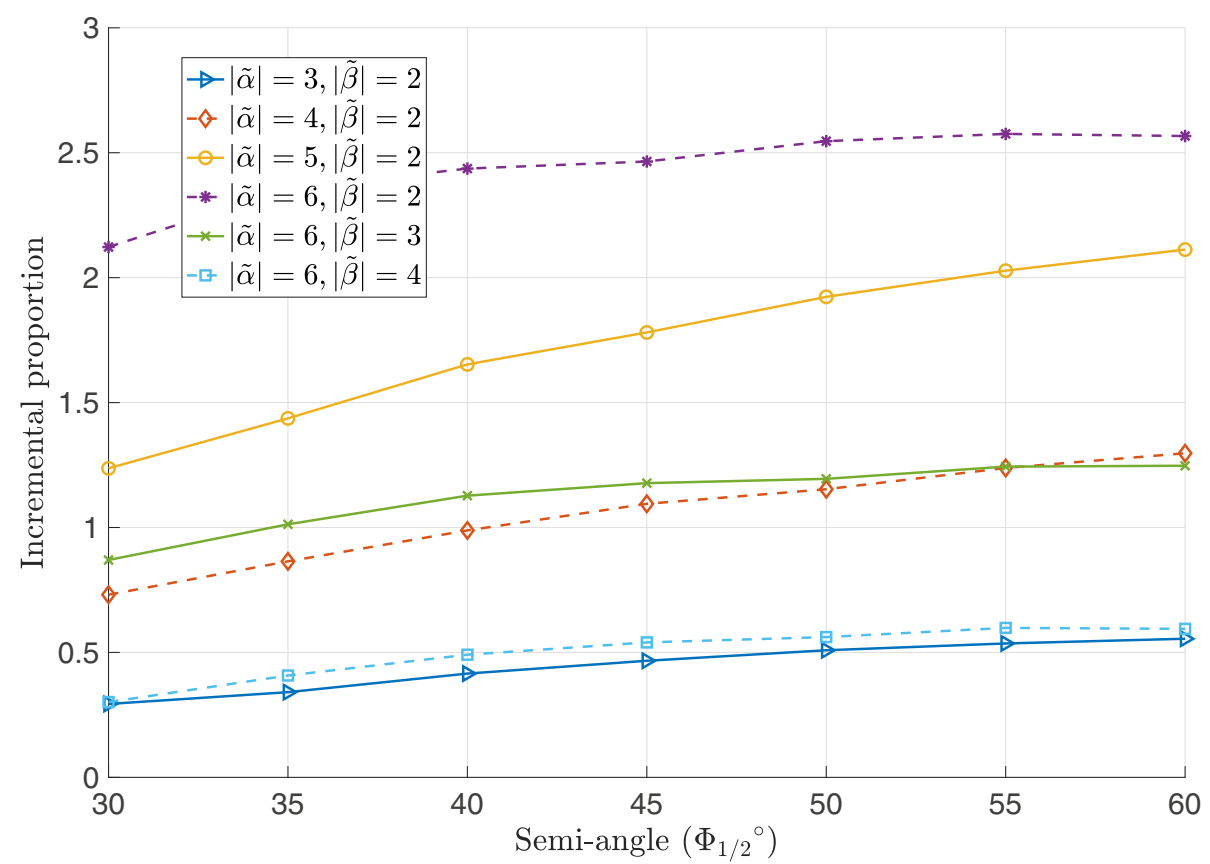

Fig. 7 Incremental proportion of VPPA with different semi-angles

access, the aforementioned derivation shows the sufficient condition, such that the users supposedly connecting to the coverage with greater number of existing users have a worse quality of association.

Figure 9 shows that the probability of the expected scenario causing the edge or overlap-area users with violation of the principle of proximity access is high in the situation that two difference cells have a gap between the number of users in each cells, whereas the gap is not extremely large. In other words, the strategy of violation of the principle of proximity access commonly can be adopted in the user grouping scenario. As when user grouping is performed, the number of users in each group commonly is load-balanced, which is similar but not identical. On the other hand, the number of users in the simulation is great for two small cells of indoor VLC. With respect to the indoor ceiling-illumination case, the Chinese national statutory building standard stipulates

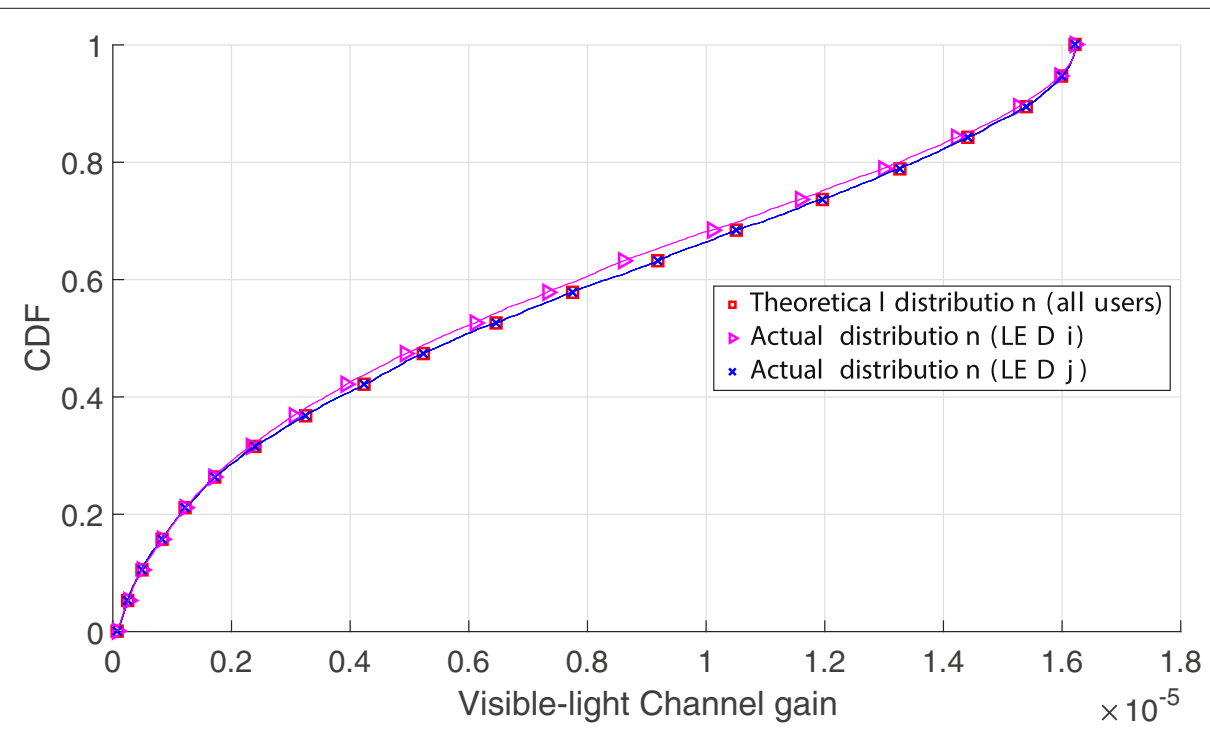

Fig. 8 CDF of multi-cell visible-light channel gains in theoretical and actual distributions 


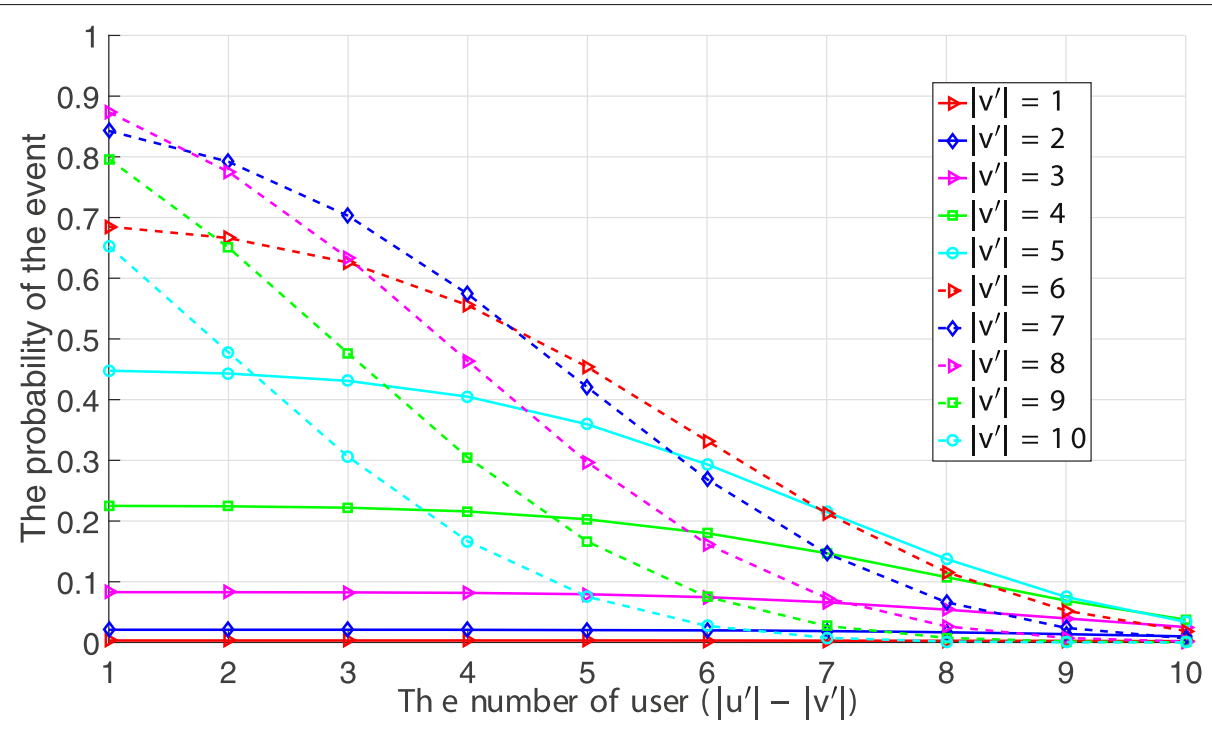

Fig. 9 Probability of the expected scenario with different number of users

that the ceiling height in citizen's home is $2.8 \mathrm{~m}$ (in GB50096-1999 and GB50096-2003), ceiling height in student apartment is not less than $3.0 \mathrm{~m}$ (in GB500961999 and GB50096-2003), ceiling height in indoor general classroom (in GBJ99-86) is not less than $3.1 \mathrm{~m}$, ceiling height in indoor special classroom (e.g., dance classroom) (in GBJ99-86) is not less than $4.5 \mathrm{~m}$, height of ceiling in car park can be between 3.5 and 11 m (in JG) 100-2015), and height of LED in industrial building can reach $8 \mathrm{~m}$ (in GB50034-2013). Note that some aforementioned documents are Chinese national standards. Hence, in a few indoor scenarios, the number of users might be high [6], which can reach the simulation value of Fig. 9.

After we analyze the probability of the expected scenario, we can observe the details in the probability for the ratio between the channel gains. Figure 10 shows that the cumulative probability increases when the halfpower semi-angle $\Phi_{1 / 2}$ or the distance between LEDs increases, which is verified by the theoretical analysis in (16). Although the maximum cumulative probability is 0.5, the value of the probability in Fig. 10, which reflects that the situation where the strategy of user association selects the VPPA may be up to a certain scale.

\section{Conclusions}

As optimal strategies for user association in multi-cell NOMA-VLC networks are challenging to achieve [7], we have studied the strategy of user association based on the principle of proximity access and presented nonapplicable conditions for the principle of proximity access. The closed-form sufficient condition for the violation of the principle of proximity access is then deduced. Based on the sufficient condition, two-case probability has been discussed. On the one hand, the scenario probability related to the number of users and visible-light channel distribution has been derived. On the other hand, the success condition probability for a single edge user has been calculated, which reveals the probability abandoning the principle of proximity access. Furthermore, the parameters in the multi-cell deployment of NOMA-VLC network have been analyzed. Finally, the numerical results are shown to be consistent with the theoretical analysis. In the future, we will analyze power allocation in multi-cell NOMA-VLC networks and user grouping strategies.

\section{Methods}

\subsection{The aim, design, and setting of the study}

The study aims for the research of user association performance in the domain of optical wireless communication (based on visible light). All verification and simulation of study are performed by the computer program.

\subsection{The characteristics of participants or description of materials}

Not applicable.

\subsection{A clear description of all processes and methodologies employed}

First, we use the mathematical model of Shannon theory to describe the basic system model. Second, we use the sufficient and necessary conditions of mathematical principles to derive the condition based on the violation of principle of proximity access. Third, we use the calculus to yield the two kinds of probability including the scenario probability and the probability of channel gain 


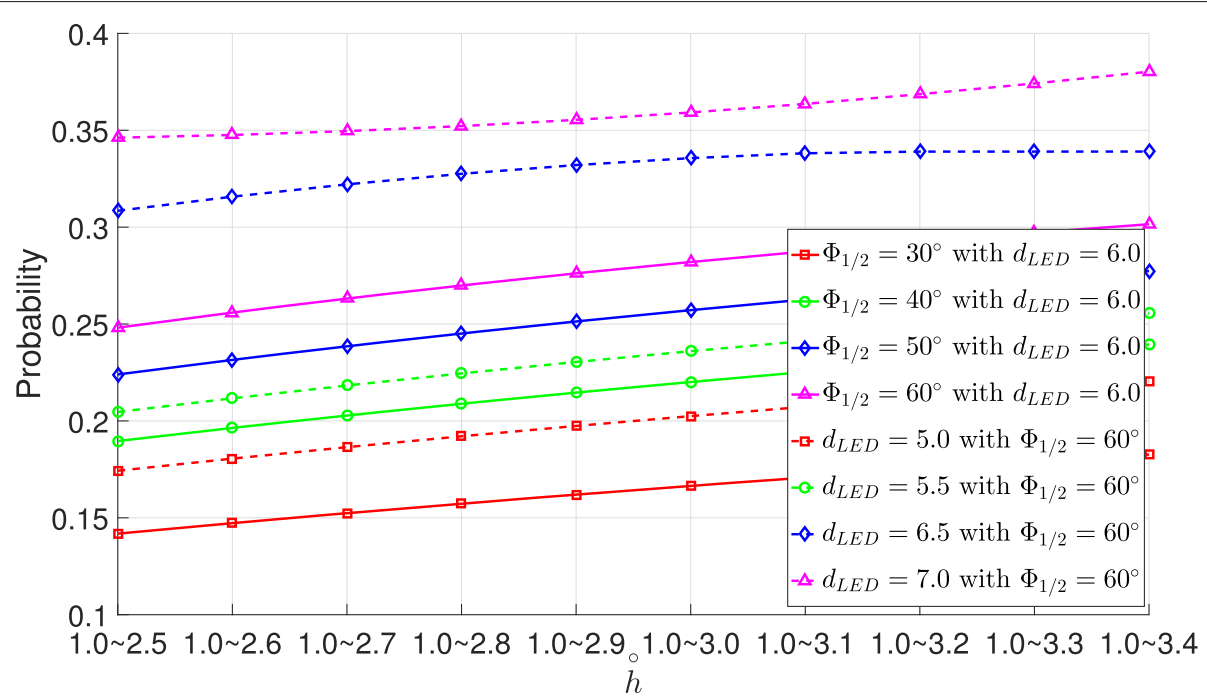

Fig. 10 Probability of the ratio between channel gains with different $\Phi_{1 / 2}$ and $d_{L E D}$

ratio. Finally, we use the Monte Carlo simulation methods and MATLAB program software to verify the theoretical analysis.

\subsection{The type of statistical analysis used, including a power calculation if appropriate}

Not applicable.

\subsection{Studies involving human participants, data, or tissue or animals must include statement on ethics approval and consent}

Not applicable. Our research is not medical or biochemistry study, and this paper is not involved with science ethical research.

\section{Abbreviations}

AP: Access point; AUDR: Average user data rate; CDF: Cumulative distribution function; CSI: Channel state information; DCS: Dynamic cell selection; FOV: Field of vision; ICl: Inter-cell interference; JT: Joint transmission; LED: Light emitting diode; LOS: Line of sight; MUI: Multi-user interference; PDF: Probability density function; PD-NOMA: Power-domain non-orthogonal multiple access; NOMA: Non-orthogonal multiple access; OMA: Orthogonal multiple access; PPA: Principle of proximity access; RF: Radio frequency; SINR: Signal-to-interference noise ratio; VLC: Visible light communication; VPPA: Violation of the principle of proximity access

\section{Acknowledgements}

This work was supported by the National Natural Science Foundation of China (nos. 61671477 and 61601516). Thanks to Dr. Yanqun Tang for technical support. The part work of this paper was presented in part at the IEEE 19th International Conference on Communication Technology (ICCT 2019), China. With the authorization from the editor, this paper has been revised and supplemented by the contributions (e.g. the probability analysis in both the expected scenario and channel gain ratio) for publication. We feel grateful for editors of ICCT 2019 and the special issue titled advanced networking technologies for next generation wireless networks.

\section{Authors' contributions}

We authors proposed the scheme of this paper. ST conducted the detailed derivations associated with performance analysis. HY, QL, and YT provide the indispensible technical suggestion and writing tips for this paper. All authors have read and approved the final manuscript.

\section{Authors' information}

Siyu tao Tao received his B.S. degree from the Beijing Institute of Technology in 2013. From 2013 to 2016, he pursued an M.S. degree from the National Digital Switching System Engineering and Technological Center (NDSC). He is currently studying for his doctorate at the NDSC. His research interests include visible light communication networking and network protocol reverse engineering. Hongyi yu Yu received his Ph.D. from Xidian University in 1998. He is currently a professor at the NDSC, and his research interests include visible light communication, wireless communication and signal processing. Qing li Li received her M.S. degree from the NDSC in 2003 and her Ph.D. from the NDSC in 2009. She is currently an associate professor working on visible light communication networking and network protocol reverse engineering. Yanqun tang Tang received his Ph.D. from the National University of Defense Technology in 2013. From 2013 to 2018, he is a university lecturer at the NDSC working on the physical layer security of wireless communication and visible light communication. Currently, he is an assistant professor in Sun Yat-sen University.

\section{Funding}

National Natural Science Foundation of China (nos. 61671477 and 61601516).

\section{Availability of data and materials}

Not applicable.

\section{Competing interests}

The authors declare that they have no competing interests.

Received: 14 November 2019 Accepted: 6 March 2020

Published online: 22 April 2020

\section{References}

1. H. Haas, L. Yin, Y. Wang, C. Chen, What is lifi? J. Lightwave Technol. 34(6), 1533-1544 (2015)

2. L. Yin, W. O. Popoola, X. Wu, H. Haas, Performance evaluation of non-orthogonal multiple access in visible light communication. IEEE Trans. Commun. 64(12), 5162-5175 (2016)

3. L. Yin, X. Wu, H. Haas, in 2015 IEEE 26th Annual International Symposium on Personal, Indoor, and Mobile Radio Communications (PIMRC). On the performance of non-orthogonal multiple access in visible light communication (IEEE, 2015), pp. 1354-1359. https://doi.org/10.1109/ pimrc.2015.7343509

4. Y. Saito, Y. Kishiyama, A. Benjebbour, T. Nakamura, A. Li, K. Higuchi, in 2013 IEEE 77th Vehicular Technology Conference (VTC Spring). Non-orthogonal multiple access (noma) for cellular future radio access (IEEE, 2013), pp. 1-5. https://doi.org/10.1109/vtcspring.2013.6692652 
5. A. Benjebbour, Y. Saito, Y. Kishiyama, A. Li, A. Harada, T. Nakamura, in 2013 International Symposium on Intelligent Signal Processing and Communication Systems. Concept and practical considerations of non-orthogonal multiple access (noma) for future radio access (IEEE, 2013), pp. 770-774. https://doi.org/10.1109/ispacs.2013.6704653

6. S. Chen, F. Qin, B. Hu, X. Li, Z. Chen, User-centric ultra-dense networks for $5 \mathrm{~g}$ : challenges, methodologies, and directions. IEEE Wirel. Commun. 23(2), 78-85 (2018)

7. W. Shin, M. Vaezi, B. Lee, D. J. Love, J. Lee, H. V. Poor, Non-orthogonal multiple access in multi-cell networks: Theory, performance, and practical challenges. IEEE Commun. Mag. 55(10), 176-183 (2017)

8. X. Li, R. Zhang, J. Wang, L. Hanzo, in IEEE International Conference on Communications. Cell-centric and user-centric multi-user scheduling in visible light communication aided networks, (2015), pp. 5120-5125. https://doi.org/10.1109/icc.2015.7249136

9. M. Moltafet, N. Mokari, M. R. Javan, H. Saeedi, H. Pishro-Nik, A new multiple access technique for $5 \mathrm{~g}$ : Power domain sparse code multiple access (psma). IEEE Access. 6, 747-759 (2017)

10. X. Guan, Q. Yang, C.-K. Chan, Joint detection of visible light communication signals under non-orthogonal multiple access. IEEE Photonics Technol. Lett. 29(4), 377-380 (2017)

11. Z. Yang, C. Pan, W. Xu, Y. Pan, M. Chen, M. Elkashlan, Power control for multi-cell networks with non-orthogonal multiple access. IEEE Trans. Wirel. Commun. 17(2), 927-942 (2017)

12. Z. Yang, W. Xu, Y. Li, Fair non-orthogonal multiple access for visible light communication downlinks. IEEE Wirel. Commun. Lett. 6(1), 66-69 (2016)

13. Y. Fu, Y. Chen, C. W. Sung, Distributed power control for the downlink of multi-cell noma systems. IEEE Trans. Wirel. Commun. 16(9), 6207-6220 (2017)

14. X. Zhang, Q. Gao, C. Gong, Z. Xu, User grouping and power allocation for noma visible light communication multi-cell networks. IEEE Commun. Lett. 21(4), 777-780 (2016)

15. M. Obeed, A. M. Salhab, M.-S. Alouini, S. A. Zummo, On optimizing vlc networks for downlink multi-user transmission: A survey. IEEE Commun. Surv. Tutor. (Early Access) (2019). https://doi.org/10.1109/comst.2019. 2906225

16. S. S. Bawazir, P. C. Sofotasios, S. Muhaidat, Y. Al-Hammadi, G. K. Karagiannidis, Multiple access for visible light communications: Research challenges and future trends. IEEE Access. 6(99), 26167-26174 (2018)

17. X. Li, F. Jin, R. Zhang, L. Hanzo, in 2016 IEEE Global Communications Conference (GLOBECOM). Joint cluster formation and user association under delay guarantees in visible-light networks (IEEE, 2016), pp. 1-6. https://doi.org/10.1109/glocom.2016.7841925

18. H. Marshoud, S. Muhaidat, P. C. Sofotasios, S. Hussain, M. A. Imran, B. S. Sharif, Optical non-orthogonal multiple access for visible light communication. IEEE Wirel. Commun. 25(2), 82-88 (2018)

19. Y. Chen, A. E. Kelly, J. H. Marsh, Improvement of indoor vlc network downlink scheduling and resource allocation. Optics Express. 24(23), 26838-26850 (2016)

20. H. Marshoud, V. M. Kapinas, G. K. Karagiannidis, S. Muhaidat, Non-orthogonal multiple access for visible light communications. IEEE Photonics Technol. Lett. 28(1), 51-54 (2015)

21. C. Shen, S. Lou, C. Gong, Z. Xu, in 2016 Annual Conference on Information Science and Systems (CISS). User association with lighting constraints in visible light communication systems (IEEE, 2016), pp. 222-227. https:// doi.org/10.1109/ciss.2016.7460505

22. X. Zhang, J. Duan, Y. Fu, A. Shi, Theoretical accuracy analysis of indoor visible light communication positioning system based on received signal strength indicator. J. Lightwave Technol. 32(21), 3578-3584 (2014)

23. R. Zhang, J. Wang, Z. Wang, Z. Xu, C. Zhao, L. Hanzo, Visible light communications in heterogeneous networks: Paving the way for user-centric design. IEEE Wirel. Commun. 22(2), 8-16 (2015)

24. R. Jiang, Q. Wang, H. Haas, Z. Wang, Joint user association and power allocation for cell-free visible light communication networks. IEEE J. Sel. Areas Commun. 36(1), 136-148 (2017)

25. X. Liu, Y. Wang, F. Zhouz, R. Q. Hu, in 2018 10th International Conference on Wireless Communications and Signal Processing (WCSP). Ber analysis for noma-enabled visible light communication systems with m-psk (IEEE, 2018), pp. 1-7. https://doi.org/10.1109/wcsp.2018.8555627
26. J. Zhao, T. Q. Quek, Z. Lei, Coordinated multipoint transmission with limited backhaul data transfer. IEEE Trans. Wirel. Commun. 12(6), 2762-2775 (2013)

27. J. Wildman, Y. Osmanlioglu, S. Weber, A. Shokoufandeh, in 2015 IEEE International Conference on Communications (ICC). Delay minimizing user association in cellular networks via hierarchically well-separated trees (IEEE, 2015), pp. 4005-4011. https://doi.org/10.1109/icc.2015.7248950

28. K. Son, H. Kim, Y. Yi, B. Krishnamachari, Base station operation and user association mechanisms for energy-delay tradeoffs in green cellular networks. IEEE J. Sel. Areas Commun. 29(8), 1525-1536 (2011)

29. D. Liu, L. Wang, Y. Chen, M. Elkashlan, K.-K. Wong, R. Schober, L. Hanzo, User association in $5 \mathrm{~g}$ networks: A survey and an outlook. IEEE Commun. Surv. Tutor. 18(2), 1018-1044 (2016)

30. S. Luo, R. Zhang, T. J. Lim, Downlink and uplink energy minimization through user association and beamforming in c-ran. IEEE Trans. Wirel. Commun. 14(1), 494-508 (2014)

31. T. Komine, M. Nakagawa, Fundamental analysis for visible-light communication system using led lights. IEEE Trans. Consumer Electron. 50(1), 100-107 (2004)

32. F. Wang, W. Chen, H. Tang, Q. Wu, Joint optimization of user association, subchannel allocation, and power allocation in multi-cell multi-association ofdma heterogeneous networks. IEEE Trans. Commun. 65(6), 2672-2684 (2017)

\section{Publisher's Note}

Springer Nature remains neutral with regard to jurisdictional claims in published maps and institutional affiliations.

\section{Submit your manuscript to a SpringerOpen ${ }^{\circ}$ journal and benefit from:}

- Convenient online submission

- Rigorous peer review

- Open access: articles freely available online

- High visibility within the field

- Retaining the copyright to your article

Submit your next manuscript at $\boldsymbol{~ s p r i n g e r o p e n . c o m ~}$ 